\title{
Full-length genome characterization and phylogenetic analysis of SARS-CoV-2 virus strains from Yogyakarta and Central Java, Indonesia
}

\author{
. Gunadi ${ }^{\text {Corresp., } 1}$, Hendra Wibawa ${ }^{\text {Corresp., }}{ }^{2}$, . Marcellus ${ }^{1}$, Mohamad Saifudin Hakim ${ }^{3}$, Edwin Widyanto Daniwijaya ${ }^{4}$, \\ Ludhang Pradipta Rizki ${ }^{3}$, Endah Supriyati ${ }^{5}$, Dwi Aris Agung Nugrahaningsih ${ }^{6}$, . Afiahayati ${ }^{7}$, . Siswanto ${ }^{8}$, Kristy \\ Iskandar ${ }^{9}$, Nungki Anggorowati ${ }^{10}$, Alvin Santoso Kalim ${ }^{1}$, Dyah Ayu Puspitarani ${ }^{1}$, Kemala Athollah ${ }^{1}$, Eggi Arguni $^{11}$ \\ , Titik Nuryastuti ${ }^{3}$, Tri Wibawa ${ }^{3}$ \\ ${ }^{1}$ Pediatric Surgery Division, Department of Surgery, Faculty of Medicine, Public Health and Nursing, Universitas Gadjah Mada, Yogyakarta, Indonesia \\ 2 Disease Investigation Center, Wates, Ministry of Agriculture, Yogyakarta, Indonesia \\ 3 Department of Microbiology, Faculty of Medicine, Public Health and Nursing, Universitas Gadjah Mada, Yogyakarta, Indonesia \\ 4 Department of Microbiology, Faculty of Medicine, Public Health and Nursing, Universitas Gadjah Mada /UGM Academic Hospital, Yogyakarta, Indonesia \\ 5 Centre of Tropical Medicine, Faculty of Medicine, Public Health and Nursing, Universitas Gadjah Mada, Yogyakarta, Indonesia \\ 6 Department of Pharmacology and Therapy, Faculty of Medicine, Public Health and Nursing, Universitas Gadjah Mada, Yogyakarta, Indonesia \\ 7 Department of Computer Science and Electronics, Faculty of Mathematics and Natural Sciences, Universitas Gadjah Mada, Yogyakarta, Indonesia \\ 8 Department of Physiology, Faculty of Medicine, Public Health and Nursing, Universitas Gadjah Mada/UGM Academic Hospital, Yogyakarta, Indonesia \\ 9 Department of Child Health, Faculty of Medicine, Public Health and Nursing, Universitas Gadjah Mada/ UGM Academic Hospital, Yogyakarta, Indonesia \\ 10 Department of Anatomical Pathology, Faculty of Medicine, Public Health and Nursing, Universitas Gadjah Mada, Yogyakarta, Indonesia \\ 11 Department of Child Health, Faculty of Medicine, Public Health and Nursing, Universitas Gadjah Mada, Yogyakarta, Indonesia \\ Corresponding Authors: . Gunadi, Hendra Wibawa \\ Email address: drgunadi@ugm.ac.id, hendra.wibawa@pertanian.go.id
}

\section{Background}

Recently, SARS-CoV-2 virus with the D614G mutation has become a public concern due to rapid dissemination of this variant across many countries. Our study aims were 1) to report full-length genome sequences of SARS-CoV-2 collected from four COVID-19 patients in the Special Region of Yogyakarta and Central Java provinces, Indonesia; 2) to compare the clade distribution of full-length genome sequences from Indonesia $(n=60)$ from March to September 2020; and 3) to perform phylogenetic analysis of SARSCoV-2 complete genomes from different countries, including Indonesia.

\section{Methods}

Whole genome sequencing (WGS) was performed using next-generation sequencing (NGS) applied in the Illumina MiSeq instrument. Full-length virus genomes were annotated using the reference genome of hCoV-19/Wuhan/Hu-1/2019 (NC_045512.2) and then visualized in UGENE v. 1.30. For phylogenetic analysis, a dataset of 88 available SARS-CoV-2 complete genomes from different countries, including Indonesia, was retrieved from GISAID.

\section{Results}

All patients were hospitalized with various severities of COVID-19. Phylogenetic analysis revealed that one and three virus samples belong to clade $\mathrm{L}$ and $\mathrm{GH}$. These three clade $\mathrm{GH}$ virus samples (EPI ISI 525492, 516800 and 516829) were not only located in a cluster with SARS-CoV-2 genomes from Peer) reviewing PDF | (2020:09:53052:2:0:NEW 19 Nov 2020) 
Asia but also those from Europe, whereas the clade L virus sample (EPI_ISL_516806) was located amongst SARS-CoV-2 genomes from Asia. Using full-length sequences available in the GISAID EpiCoV Database, 39 of 60 SARS-CoV-2 (65\%) from Indonesia harbor the D614G mutation.

\section{Conclusion}

These findings indicate that SARS-CoV-2 with the D614G mutation appears to become the major circulating virus in Indonesia, concurrent with the COVID-19 situation worldwide. 
1 Full-length genome characterization and phylogenetic

2 analysis of SARS-CoV-2 virus strains from Yogyakarta

3 and Central Java, Indonesia

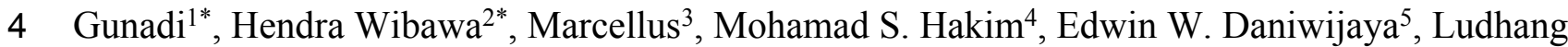

5 P. Rizki ${ }^{6}$, Endah Supriyati ${ }^{7}$, Dwi AA. Nugrahaningsih ${ }^{8}$, Afiahayati ${ }^{9}$, Siswanto ${ }^{10}$, Kristy

6 Iskandar ${ }^{11}$, Nungki Anggorowati ${ }^{12}$, Alvin S. Kalim ${ }^{13}$, Dyah Ayu Puspitarani ${ }^{14}$, Kemala

7 Athollah $^{15}$, Eggi Arguni ${ }^{16}$, Titik Nuryastuti ${ }^{17}$, Tri Wibawa ${ }^{18}$, on behalf of the Yogyakarta-Central

8 Java COVID-19 study group

9

10

11

12

13

14

15

16

17

18

19

20

21

22

23

24

25

26

27

28

29

30

31

32

33

34

35

36

37

38

39

40

${ }^{1}$ Pediatric Surgery Division, Department of Surgery, Faculty of Medicine, Public Health and Nursing, Universitas Gadjah Mada, Yogyakarta, Indonesia; drgunadi@ugm.ac.id

${ }^{2}$ Disease Investigation Center, Wates, Yogyakarta, Ministry of Agriculture Indonesia; hendra.wibawa@pertanian.go.id

${ }^{3}$ Pediatric Surgery Division, Department of Surgery, Faculty of Medicine, Public Health and Nursing, Universitas Gadjah Mada, Yogyakarta, Indonesia; marcelluskorompis@gmail.com

${ }^{4}$ Department of Microbiology, Faculty of Medicine, Public Health and Nursing, Universitas Gadjah Mada, Yogyakarta, Indonesia; m.s.hakim@ugm.ac.id

${ }^{5}$ Department of Microbiology, Faculty of Medicine, Public Health and Nursing, Universitas Gadjah Mada /UGM Academic Hospital, Yogyakarta, Indonesia; m.edwin.d@ugm.ac.id ${ }^{6}$ Department of Microbiology, Faculty of Medicine, Public Health and Nursing, Universitas Gadjah Mada, Yogyakarta, Indonesia; ludhang@ugm.ac.id

${ }^{7}$ Centre of Tropical Medicine, Faculty of Medicine, Public Health and Nursing, Universitas Gadjah Mada, Yogyakarta, Indonesia; endah.supriyati@worldmosquito.org

${ }^{8}$ Department of Pharmacology and Therapy, Faculty of Medicine, Public Health and Nursing, Universitas Gadjah Mada, Yogyakarta, Indonesia; dwi.aris.a@ugm.ac.id

${ }^{9}$ Department of Computer Science and Electronics Faculty of Mathematics and Natural Sciences, Universitas Gadjah Mada, Yogyakarta, Indonesia; afia@ugm.ac.id ${ }^{10}$ Department of Physiology, Faculty of Medicine, Public Health and Nursing, Universitas Gadjah Mada/UGM Academic Hospital, Yogyakarta, Indonesia; sis12@ugm.ac.id ${ }^{11}$ Department of Child Health, Faculty of Medicine, Public Health and Nursing, Universitas Gadjah Mada/ UGM Academic Hospital, Yogyakarta, Indonesia; kristy.iskandar@mail.ugm.ac.id

${ }^{12}$ Department of Anatomical Pathology, Faculty of Medicine, Public Health and Nursing, Universitas Gadjah Mada, Yogyakarta, Indonesia; nungki@ugm.ac.id

${ }^{13}$ Pediatric Surgery Division, Department of Surgery, Faculty of Medicine, Public Health and Nursing, Universitas Gadjah Mada, Yogyakarta, Indonesia; alvin_2495@yahoo.com

${ }^{14}$ Pediatric Surgery Division, Department of Surgery, Faculty of Medicine, Public Health and Nursing, Universitas Gadjah Mada, Yogyakarta, Indonesia; raden.ajeng.d@mail.ugm.ac.id ${ }^{15}$ Pediatric Surgery Division, Department of Surgery, Faculty of Medicine, Public Health and Nursing, Universitas Gadjah Mada, Yogyakarta, Indonesia; kemala.athollah@mail.ugm.ac.id 
$41{ }^{16}$ Department of Child Health, Faculty of Medicine, Public Health and Nursing, Universitas

42 Gadjah Mada, Yogyakarta, Indonesia; eggiarguni@ugm.ac.id

$43{ }^{17}$ Department of Microbiology, Faculty of Medicine, Public Health and Nursing, Universitas

44 Gadjah Mada, Yogyakarta, Indonesia; t.nuryastuti@ugm.ac.id

$45{ }^{18}$ Department of Microbiology, Faculty of Medicine, Public Health and Nursing, Universitas

46 Gadjah Mada, Yogyakarta, Indonesia; twibawa@ugm.ac.id

47

48 Corresponding Author:

49 Gunadi $^{1}$

50 Faculty of Medicine, Public Health and Nursing, Universitas Gadjah Mada/Dr. Sardjito Hospital

51 Jl. Kesehatan No. 1 Yogyakarta 55281, Indonesia

52 drgunadi@ugm.ac.id

53

54

Hendra Wibawa ${ }^{2}$

55

Wates Disease Investigation Center

Jl. Raya Wates Km. 27, Kulon Progo, Yogyakarta, Indonesia 55651

57

hendra.wibawa@pertanian.go.id

\section{Abstract}

Background: Recently, SARS-CoV-2 virus with the D614G mutation has become a public concern due to rapid dissemination of this variant across many countries. Our study aims were: 1) to report full-length genome sequences of SARS-CoV-2 collected from four COVID-19 patients in the Special Region of Yogyakarta and Central Java provinces, Indonesia; 2) to compare the clade distribution of full-length genome sequences from Indonesia $(n=60)$ from March to September 2020; and 3) to perform phylogenetic analysis of SARS-CoV-2 complete genomes from different countries, including Indonesia.

Methods: Whole genome sequencing was performed using next-generation sequencing applied in the Illumina MiSeq instrument. Full-length virus genomes were annotated using the reference genome of hCoV-19/Wuhan/Hu-1/2019 (NC_045512.2) and then visualized in UGENE v. 1.30. For phylogenetic analysis, a dataset of 88 available SARS-CoV-2 complete genomes from different countries, including Indonesia, was retrieved from GISAID.

72 Results: All patients were hospitalized with various severities of COVID-19. Phylogenetic analysis revealed that one and three virus samples belong to clade $\mathrm{L}$ and $\mathrm{GH}$, respectively. These three clade GH virus samples (EPI_ISL_525492, 516800 and 516829) were not only located in a cluster with SARS-CoV-2 genomes from Asia but also those from Europe, whereas the clade L virus sample (EPI_ISL_516806) was located amongst SARS-CoV-2 genomes from Asia. Using

77 full-length sequences available in the GISAID EpiCoV Database, 39 of 60 SARS-CoV-2 (65\%) from Indonesia harbor the D614G mutation. 
79 Conclusion: These findings indicate that SARS-CoV-2 with the D614G mutation appears to 80 become the major circulating virus in Indonesia, concurrent with the COVID-19 situation 81 worldwide.

82

83 Keywords: COVID-19; D614G mutation; Indonesia; phylogenetic analysis; SARS-CoV-2; 84 whole genome sequencing

85

86 


\section{Introduction}

88 In December 2019, an outbreak of Severe Acute Respiratory Syndrome Coronavirus 2 (SARS-

89 CoV-2) causing Coronavirus Disease 2019 (COVID-19) was detected in Wuhan, China and has

90 become a global pandemic, including Indonesia $[1,2]$.

91 In Indonesia, the first two COVID-19 cases were reported on March 2nd, 2020. Since then, the

92 confirmed cases have been continuously increasing although several public health measures,

93 involving isolation of confirmed patients and community wide containment in addition to strictly

94 enforced personal health protocols, were conducted to halt transmission events [3]. Tragically,

95 on November 19, 2020, Indonesia recorded 478,720 COVID-19 infections and 15,503 deaths [3].

96 This situation means that Indonesia has reported the second most confirmed COVID-19 cases in

97 the South East Asia countries after the Philippines, yet has the highest number of deaths caused

98 by COVID-19 among other South East Asia countries [3].

99 Just as many other countries, the detection of SARS-CoV-2 in suspected people is mainly based

100 on reverse transcriptase real-time polymerase chain reaction (RT-PCR). The supply of PCR

101 reagents, trained lab personnel and the availability of laboratories with sufficient biocontainment

102 levels are major challenges of SARS-CoV-2 detection in developing countries, such as Indonesia

103 [4]. Therefore, it is not surprising that the tested people per week is still lower than the World

104 Health Organization (WHO) standard [3].

105 Recently, SARS-CoV-2 with the D614G mutation became the most frequently detected globally, 106 including South East Asia region [5,6]. Interestingly, SARS-CoV-2 with the G614 variant had

107 significantly higher infectious titers than the original D614 virus, and COVID-19 patients with

108 the G614 variant had a higher viral load than patients without the mutation [5]. A recent study

109 showed that the SARS-CoV-2 with the G614 variant revealed increased infectivity, competitive

110 fitness, and transmission than the wild-type D614 virus in human airway epithelial cells and

111 hamster [7]. However, this mutation was not associated with the severity of COVID-19 [5,6].

112 Here, we aimed: 1) to report full-length genome sequences of SARS-CoV-2 collected from four

113 COVID-19 patients in the Special Region of Yogyakarta and Central Java provinces, Indonesia;

114 2) to compare the clade distribution of full-length genome sequences from Indonesia $(n=60)$

115 from March to September 2020; and 3) to perform phylogenetic analysis of SARS-CoV-2

116 complete genomes from different countries, including Indonesia. 


\section{Materials \& Methods}

\section{Classification of COVID-19 severity}

120 We determined the COVID-19 severity according to the WHO classifications: 1) mild, 121 symptomatic COVID-19 patients without evidence of hypoxia or pneumonia; 2) moderate, 122 clinical signs of pneumonia (i.e., fever, cough, dyspnea, fast breathing) but not severe 123 pneumonia, including blood oxygen saturation levels $\left(\mathrm{SpO}_{2}\right) \geq 90 \%$ in room air; 3$)$ severe, 124 clinical signs of pneumonia plus one of the conditions as follows: respiratory rate $>30$ 125 breaths/minute, severe respiratory distress, or $\mathrm{SpO}_{2}<90 \%$ in room air; and 4) critical, Acute 126 Respiratory Distress Syndrome (ARDS), sepsis, or septic shock, other complications such as 127 acute pulmonary embolism, acute coronary syndrome, acute stroke, and delirium [8].

\section{Virus samples}

All virus samples were collected from hospitalized patients with COVID-19 from June-August 2020 in Yogyakarta and Central Java provinces. Samples were collected from nasopharyngeal swabs and then directly put into viral transport media (DNA/RNA Shield ${ }^{\mathrm{TM}}$ Collection Tube with Swab, Zymo Research, CA, United States). Samples were sent to the Department of Microbiology and Laboratorium Diagnostik Yayasan Tahija World Mosquito Program, Faculty of Medicine, Public Health and Nursing, Universitas Gadjah Mada and the Disease Investigation Center, Wates, Yogyakarta for SARS-CoV-2 virus detection using Real-Q 2019-nCoV Detection Kit (BioSewoom, Seoul, South Korea) with LightCycler ${ }^{\circledR} 480$ Instrument II (Roche Diagnostics, Mannheim, Germany).

\section{Whole genome sequencing}

141 Total viral RNA was extracted from 15 original samples (nasopharyngeal swabs) using a QiAMP

142 Viral RNA mini kit (Qiagen, Hilden, Germany), followed by double stranded cDNA synthesis 143 using Maxima H Minus Double-Stranded cDNA Synthesis (Thermo Fisher Scientific, MA, 144 United States), and then purified by a GeneJET PCR Purification Kit (Thermo Fisher Scientific, 145 MA, United States). The Nextera DNA Flex for Enrichment using Respiratory Virus Oligos 146 Panel was used for library preparations, and whole genome sequencing was performed using 147 next generation sequencing (NGS) applied in the Illumina MiSeq instrument (Illumina, San 148 Diego, CA, United States) with Illumina MiSeq reagents v3 150 cycles ( 2 x 75 cycles). Among 
14915 samples that were analyzed by NGS, only four samples showed good data for further

150 bioinformatics analysis. The paired reads were trimmed for quality and length and assembled by 151 mapping to the reference genome from Wuhan, China (hCoV-19/Wuhan/Hu-1/2019, GenBank

152 accession number: NC_045512.2) using Burrow-Wheeler Aligner (BWA) algorithm embedded

153 in UGENE v. 1.30 [9]. Single nucleotide polymorphism (SNP)_was identified based on the

154 number of high confidence base calls (consensus sequence variations of the assembly) that

155 disagree with the reference bases for the genome position of interest. These variations were then

156 exported to a vef file and visualized in MS Excel. All four full-genome sequences of SARS-

157 CoV-2 had the following accession IDs: EPI_ISL_516800, EPI_ISL_516806, EPI_ISL_516829, 158 and EPI_ISL_525492 [10].

159

160

Genome annotation and phylogenetic analysis

161 Full-length virus genomes were annotated using the reference genome of $\mathrm{hCoV}-19 / \mathrm{Wuhan} / \mathrm{Hu}-$ 162 1/2019 (NC_045512.2). For phylogenetic analysis, a dataset of 88 available SARS-CoV-2 virus 163 genomes from different countries, including those from Indonesia, was retrieved from GISAID 164 (Acknowledgment Table provided in Supplementary Data). Instead of using all available 165 sequences in GISAID, we used sequences of several viruses representing SARS-CoV-2 clades 166 from some countries that have complete genome, high-coverage, and no stretches of 'NNNN' for 167 the phylogenetic dataset. Sequence alignment was performed using the MAFFT program server 168 for multiple nucleotide sequence alignment (https://mafft.cbrc.jp/alignment/server/). A 169 phylogenetic tree was constructed using 29.400 nucleotide length starting from the ORF1ab open 170 reading frame of SARS-CoV-2 using a maximum likelihood statistical method with 1,000 171 bootstrap replications and selected the best-fitting substitution model $(\mathrm{GTR}+\mathrm{G}+\mathrm{I})$ for the dataset. 172 All the analyses were performed in Molecular Evolutionary Genetics Analysis version 10 173 (MEGA X) software [11]. Because the purpose of this phylogenetic analysis was to determine 174 the evolutionary relationships between our virus samples and the other SARS-CoV-2 viruses, the 175 tree was rooted to the oldest virus, hCoV-19/Wuhan/Hu-1/2019.

Ethical Approval

178 The Medical and Health Research Ethics Committee of the Faculty of Medicine, Public Health 179 and Nursing, Universitas Gadjah Mada/Dr. Sardjito Hospital approved this study 
180 (KE/FK/0563/EC/2020). Written informed consent was obtained from all participants before 181 joining in this study.

182

183

184

Results

185

Whole genome sequences of SARS-CoV-2 from the Special Region of Yogyakarta and Central Java provinces, Indonesia

187

188

189

190

191

192

193

194

195

196

197

198

199

200

201

202

203 (Insert Tables 1-3 here)

204

205

206

207

208

209

210 (M49I) (Table 1).

All patients were classified as moderate COVID-19, except patient-4 as a mild case (Table 1 and Suppl. Table 1). The details of case presentations are described in the Supplementary Table 1. WGS revealed that the virus sample collected from patient-1 (hCoV19/Indonesia/YO-UGM781481/2020, ID: EPI_ISL_516829), patient-2 (hCoV19/Indonesia/YO-UGM-202449/2020, ID: EPI_ISL_516800), and patient-3 belonged to the GH clade, while those from patient-4 (hCoV19/Indonesia/YO-UGM-200927/2020, ID: EPI_ISL_516806) showed the L clade.

Moreover, WGS of virus from patient-1, patient-2, and patient 3 showed 9 amino acid mutations in 6 proteins, including NSP3 (P679S), NSP12 (P323L, A656S), NSP13 (M576I), spike (D614G), NS3 (A54V, Q57H, A99S), and NP (Q160R); 4 amino acid mutations in 4 proteins: NSP3 (P822L), NSP12 (P323L), Spike (D614G), and NS3 (Q57H); and 5 amino acid mutations in 5 proteins: NSP3 (P822L), NSP12 (P323L), Spike (D614G), NS3 (Q57H), and NS7a (H73Y), respectively; whereas those from patient-4 consisted of only one mutation in the NSP5 protein

The genome-wide SNPs and amino acid variations of our samples are shown in Table 2 and 3, respectively (positions referred to the reference sequence: NC_045512.2). Not all SNPs cause amino acid changes in our samples.

\section{Clade distribution of full-length genome sequences from Indonesia}

Whole genome sequencing revealed that one virus (hCoV19/Indonesia/YO-202449/2020, EPI_ISL_516800) had a complete SARS-CoV-2 genome (29.903 nt). Although the other three virus samples were shorter due to incomplete UTRs at either the 5' or 3', they possessed fulllength and complete open reading frames (ORFs) with a size of 29.409 nt consisting of 11 genes (ORF1ab, S, ORF3a, E, M, ORF6, ORF7a, ORF7b, ORF8, N, ORF10). 
211 Next, we compared the clade distribution of full-length genome sequences from

212 Indonesia $(\mathrm{n}=60)$ from March to September 2020. Based on the collection data, most $(39 / 60$, $21365 \%$ ) virus genomes contained the D614G mutation representing clade G (2), GR (7), and GH

214 (30) (Fig. 1). From March to April 2020, clade L was dominant. On the other hand, there has 215 been an increase in the detection of clade GH since April 2020 until now.

216

217 (Insert Figure 1 here)

218

219 Phylogenetic analysis

220 Phylogenetic analysis of whole genome sequencing showed that three virus samples

221 (EPI_ISL_525492,EPI_ISL_516800 and EPI_ISL_516829) clustered amongst viruses from the 222 clade GH from multiple countries across Asia, Middle East, and Europe (Fig. 2). In particular to 223 EPI_ISL_516829, this virus showing an extended branch length to the ancestral human SARS$224 \mathrm{CoV}-2$ virus, hCoV-19/Wuhan/Hu-1/2019. On the other hand, one virus sample 225 (EPI_ISL_516806) was situated between clade L viruses mainly from Asia (China, Malaysia, 226 Indonesia, India, United Arab Emirates, and Japan) (Fig. 2)

227

228 (Insert Figure 2 here)

229

\section{Discussion}

231 The present study reports four full genomes of SARS-CoV-2 from patients with COVID-19 in 232 Yogyakarta and Central Java Provinces, Indonesia. Phylogenetic analysis showed that 3 of 4 233 samples collected in June and August 2020 were clustered within SARS-CoV-2 viruses 234 belonging to GH clade. One of these (EPI_ISL_516829) displayed a longer branch length 235 compared to the other viruses indicating a greater evolutionary distance to the ancestral virus, $236 \mathrm{hCoV}-19 / \mathrm{Wuhan} / \mathrm{Hu}-1 / 2019$. This was confirmed by the molecular characterization showing that 237 more SNPs and amino acid mutations were found within this virus genome. The phylogenetic 238 analysis also revealed that one virus sample collected in May 2020 (EPI_ISL_516806) was in 239 evolution closely related to the other SARS-CoV-2 viruses belonged to the L clade, including 240 those from Indonesia which were collected in the early months of disease transmission during the 241 pandemic between March and April 2020. 
242 Our finding corresponds with the situation in Indonesia, showing that during the early pandemic 243 only two clades, $\mathrm{O}$ and $\mathrm{L}$, were detected, and the latter clade was more dominantly found from 244 COVID-19 cases. However, since the first detection of clade GH in April 2020, this virus was 245 more frequently detected than the previous circulating clades. Based on the data available in 246 GISAID, 60 virus samples representing five clades have been detected from COVID-19 cases in 247 Indonesia up to September 2020 (based on full-length genome and submission date): L (20), O 248 (1), G (2), GR (7), and GH (30) [10]. Whether this pattern correlates with the increase in the 249 number of COVID-19 cases recently in Indonesia has to be investigated further. Interestingly, a 250 similar situation was found in some countries in North America [12] and Africa [13], which also 251 detected more SARS-CoV-2 virus strains belonging to clade GH than to the other clades. An 252 increase in SARS-CoV-2 detection conveys the D614G mutation concurrent with the recent 253 global situation of COVID-19 [10].

254 The D614G mutation dominates globally approximately 77,818/96,215 ( 81\%) full genomes 255 submitted at GISAID until September 18, 2020 [10]. Three of four (75\%) SARS-CoV-2 in our 256 case series also consisted of D614G. According to phylogenetic tree and sequence distribution 257 analysis, it has been suggested that the dominating D614G globally is caused by a positive 258 selection [5], while the dominating D614G in Europe is due to a founder effect [14]. Whether 259 which mechanism occurs in Indonesia is difficult to conclude since only limited full genomes 260 were submitted to GISAID until the submission date of the end of September $2020(n=60)$ [10]. 261 The virus with the D614G mutation in Indonesia was first detected in April 2020 in Surabaya, 262 East Java [10], followed by other provinces, including Yogyakarta, Central Java, West Java and 263 Banten. Clade L was mostly detected in Jakarta (7/20) and Surabaya, East Java (7/20), followed 264 by Papua (3/20) (Fig. 1) [10].

265 It has been reported that COVID-19 patients with the D614G mutation have a higher viral load 266 than patients infected by SARS-CoV-2 without mutations [5]. The patients with D614G had a $\mathrm{C}_{\mathrm{T}}$ 267 value lower than one patient without the mutation (Table 1).

268 Interestingly, patients infected with SARS-CoV-2 bearing D614G mutations showed moderate 269 COVID-19, while the patient without mutations suffered from mild symptoms. These differences 270 might be associated with the small sample size of our study $(\mathrm{n}=4)$ compared with previous 271 studies ( $\mathrm{n}=999$ [5], 175 [15], and 88 [16]). Moreover, the severity of COVID-19 is affected by 272 many factors, including age, sex, presence of comorbidities, and patients' immune responses 
$273[8,17]$. Further study with a larger sample size and involving risk factors for COVID-19 severity

274 is mandatory to determine the association between the D614G mutation and the severity of

275 COVID-19, particularly in Indonesia.

276 Among GH clades, they also consisted of different mutations in addition to the variants that

277 determine the clade name (Table 1). It has already been reported that the D614G variant is

278 almost always accompanied by three other variants: a C-to-T change in the 5'UTR, a silent

279 c.3307C $>$ T variant, and P323L [4]. All GH clade samples in the present study also contained 280 P323L (Table 1).

281 Notably, whole genome sequencing is of practical importance to determine virus variants and

282 clades and is associated with particular geographic disseminations to decide clinical and political

283 approaches at the regional and local levels [12]. Moreover, whether the differences in the case

284 fatality rate and viral spread or transmission among different countries/regions are affected by

285 differences in the virus clade [18] needs to be further studied.

286 Our study has some limitations, including that the samples sequenced are only $0.02 \%$

$287(60 / 290,000)$ of all confirmed cases in Indonesia. These facts should be considered in the 288 interpretations of our findings, especially about epidemiological patterns (e.g. increase vs.

289 decrease of frequency) of a particular clade in Indonesia. Another limitation of our study is we

290 do not have any data from epidemiological tracing, nor data concerning detection (how far, how

291 fast) to determine what is the size of the cluster where each patient belongs, or what is the

292 transmission pattern of the cluster.

293

294 Conclusions

295 We report the full-genome sequence characterization and phylogenetic analysis of SARS-CoV-2

296 from Indonesia. SARS-CoV-2 with the D614G mutation appears to become the major circulating

297 virus in Indonesia, which is concurrent with the COVID-19 situation worldwide. Further study

298 with a larger sample size is necessary to investigate whether the dominating SARS-CoV-2

299 bearing the D614G mutation is due to a positive selection or a founder effect or some other

300 mechanism and to explore the role of the D614G mutation in the pathogenesis and virulence of

301 SARS-CoV-2.

302

303 Acknowledgements 
304 We thank the Collaborator Members of the Yogyakarta-Central Java COVID-19 study group: 305 Kurniyanto (RSUP Dr. Soeradji Tirtonegoro), Indah Juliana (RSUP Dr Soeradji Tirtonegoro), 306 Beby Dewi Sartika (RSUD Nyi Ageng Serang), Ardorisye Saptaty Fornia (RS PKU Gamping), 307 Dwiki Afandy (Faculty of Medicine, Public Health and Nursing, Universitas Gadjah Mada (FK308 KMK UGM)), Susan Simanjaya (FK-KMK UGM), William Widitjiarso (FK-KMK UGM), 309 Aditya Rifqi Fauzi (FK-KMK UGM), Safitriani (PT. Pandu Biosains), Muhammad Taufiq 310 Soekarno (PT. Pandu Biosains) and Sri Fatmawati (FK-KMK UGM). Gunadi, Marcellus, Dwi 311 Aris Agung Nugrahaningsih, Kristy Iskandar, Nungki Anggorowati, Alvin Santoso Kalim, 312 Kemala Athollah, Dyah Ayu Puspitarani are members of the Genetics Working Group (Pokja

313 Genetik), Faculty of Medicine, Public Health and Nursing, Universitas Gadjah Mada. We 314 gratefully acknowledge the authors, the Originating and Submitting Laboratories for their 315 sequence and metadata shared through GISAID. All submitters of data may be contacted directly 316 via www.gisaid.org. The Acknowledgments Table for GISAID is reported as Supplementary 317 material.

318

\section{References}

320

321

322

323

324

325

326

327

328

329

330

331

332

333

334

335

336

337

338

339

340

341

342

1. World Health Organization. https://www.who.int/news-room/detail/27-04-2020-whotimeline---covid-19 Accessed on June 14, 2020.

2. Phelan AL, Katz R, Gostin LO. The novel coronavirus originating in Wuhan, China: challenges for global health governance. JAMA. 2020 323, 709-710, doi:10.1001/jama.2020.1097

3. World Health Organization. https://covid19.who.int/table Accessed on October 10, 2020.

4. Younes N, Al-Sadeq DW, Al-Jighefee H, Younes S, Al-Jamal O, Daas HI, Yassine HM, Nasrallah GK. Challenges in laboratory diagnosis of the novel Coronavirus SARS-CoV-2. Viruses. 2020; 26;12:582.

5. Korber B, Fischer WM, Gnanakaran S, Yoon H, Theiler J, Abfalterer W, Hengartner N, Giorgi E, Bhattacharya T, Foley B, Hastie K, Parker M, Partridge D, Evans C, Freeman T, de Silva T. Tracking changes in SARS-CoV-2 spike: evidence that D614G increases infectivity of the COVID-19 virus. Cell 2020, 182, 812-827, doi: 10.1016/j.cell.2020.06.043

6. Nguyen TT, Pham TN, Van TD, Nguyen TT, Nguyen DTN, Le HNM, Eden JS, Rockett RJ, Nguyen TTH, Vu BTN, Tran GV, Le TV, Dwyer DE, van Doorn HR; OUCRU COVID-19 Research Group. Genetic diversity of SARS-CoV-2 and clinical, epidemiological characteristics of COVID-19 patients in Hanoi, Vietnam. PLoS One. 2020 Nov 17;15(11):e0242537.

7. Hou YJ, Chiba S, Halfmann P, Ehre C, Kuroda M, Dinnon KH 3rd, Leist SR, Schäfer A, Nakajima N, Takahashi K, Lee RE, Mascenik TM, Graham R, Edwards CE, Tse LV, Okuda K, Markmann AJ, Bartelt L, de Silva A, Margolis DM, Boucher RC, Randell SH, Suzuki T, Gralinski LE, Kawaoka Y, Baric RS. SARS-CoV-2 D614G variant exhibits efficient replication ex vivo and transmission in vivo. Science. 2020 Nov 12:eabe8499. 
343 8. Beeching NJ, Fletcher TE, Fowler R. BMJ best practice. Coronavirus Disease 2019 (COVID19). https://bestpractice.bmj.com/topics/en-us/3000168/prognosis Accessed on October 10, 2020.

346

347

348

349

350

351

352

353

354

355

356

357

358

359

360

361

362

363

364

365

366

367

368

369

370

371

372

373

374

375

376

9. https://ugene.net/wiki/display/UUOUM30/About+UGENE Accessed on August 20, 2020.

10. GISAID. https://platform.gisaid.org/epi3/cfrontend\#8dc5e

11. Kumar S. Stecher G, Li M, Knyaz C, Tamura K. MEGA X: Molecular Evolutionary Genetics Analysis across computing platforms. Mol Biol Evol. 2018;35:1547-1549.

12. Mercatelli D, Giorgi FM. Geographic and genomic distribution of SARS-CoV-2 Mutations. Front Microbiol 2020, 11, 1800, doi: 10.3389/fmicb.2020.01800

13. Wruck W, Adjaye J. Transmission of SARS-COV-2 from China to Europe and West Africa: a detailed phylogenetic analysis. bioRxiv preprint. doi: https://doi.org/10.1101/2020.10.02.323519.

14. Dearlove B, Lewitus E, Bai H, Li Y, Reeves DB, Joyce MG, Scott PT, Amare MF, Vasan S, Michael NL, Modjarrad K, Rolland M. A SARS-CoV-2 vaccine candidate would likely match all currently circulating variants. Proc Natl Acad Sci USA 2020, 202008281, doi: 10.1073/pnas.2008281117

15. Wagner C, Roychoudhury P, Frazar C, Lee J, Muller N, Moncla L, Hadfield J, Hodcroft E, Pelle B, Richardson M, Behrens C, Huang M, Mathias P, Pepper G, Shrestha L, Xie H, Addetia A, Nguyen T, Rachleff V, Gautom R, Melly G, Hiatt B, Dykema P, Adler A, Brandsetter E, Han P, Fay K, Ilcsin M, Lacombe K, Sibley T, Tryong M, Wolf C, Cowgill K, Schrag S, Duchin J, Boeckh M, Englund J, Famulare M, Lutz B, Rieder M, Thompson M, Neher R, Baird G, Starita L, Chu H, Shendure J, Lindquist S, Nickerson D, Greninger A, Jerome K, Bedford T. Comparing viral load and clinical outcomes in Washington State across D614G mutation in spike protein of SARS-CoV-2. 2020; https://github.com/blab/ncov-D614G

16. Lorenzo-Redondo R, Nam HH, Roberts SC, Simons LM, Jennings LJ, Qi C, Achenbach CJ, Hauser AR, Ison MG, Hulquist JF, Ozer EA. A unique clade of SARS-CoV-2 viruses is associated with lower viral loads in patient upper airways. Preprint. medRxiv 2020, 2020.05.19.20107144, doi: 10.1101/2020.05.19.20107144

17. Zou L, Dai L, Zhang Y, Fu W, Gao Y, Zhang Z, Zhang Z. Clinical characteristics and risk factors for disease severity and death in patients with Coronavirus Disease 2019 in Wuhan, China. Front Med (Lausanne). 2020;7:532.

18. Brufsky A. Distinct viral clades of SARS-CoV-2: implications for modeling of viral spread. J. Med. Virol 2020, doi: 10.1002/jmv.25902. 


\section{Figure Titles and Legends}

378 Figure 1. Clade distribution of SARS-CoV-2 genomes in Indonesia until the submission date of 379 September 2020, showing that $65 \%$ contained the D614G mutation. All G clades (G, GH and 380 GR) carried the D614G mutation.

381

382 Figure 2. Phylogenetic analysis of SARS-CoV-2 genomes from Indonesia and different 383 countries. A phylogenetic tree was constructed from $29.400 \mathrm{nt}$ length of the open reading frame 384 (ORF) of SARS-CoV-2 using the maximum likelihood statistical method with 1,000 bootstrap 385 replications and best-fitting DNA substitution model $(\mathrm{GTR}+\mathrm{G}+\mathrm{I})$ for the dataset. Virus samples 386 from Yogyakarta and Central Java are indicated in red, while those from other regions in 387 Indonesia are indicated in blue. The tree is drawn to scale (0.0001), with branch lengths 388 measured in the number of substitutions per site.

389

390 Table List

391 Table 1. Characteristics of four patients with COVID-19 and SARS-CoV-2 virus samples from 392 Yogyakarta and Central Java.

393 Table 2. The genome-wide SNPs of four SARS-CoV-2 virus samples from Yogyakarta and 394 Central Java (positions referred to the reference sequence: NC_045512.2).

395 Table 3. The amino acid variations of four SARS-CoV-2 virus samples from Yogyakarta and 396 Central Java (positions referred to the reference sequence: NC_045512.2).

397 
Figure 1

Clade distribution of SARS-CoV-2 genomes in Indonesia until the submission date of September 2020 , showing that $65 \%$ contained the D614G mutation. All G clades (G, GH and GR) carried the D614G mutation.

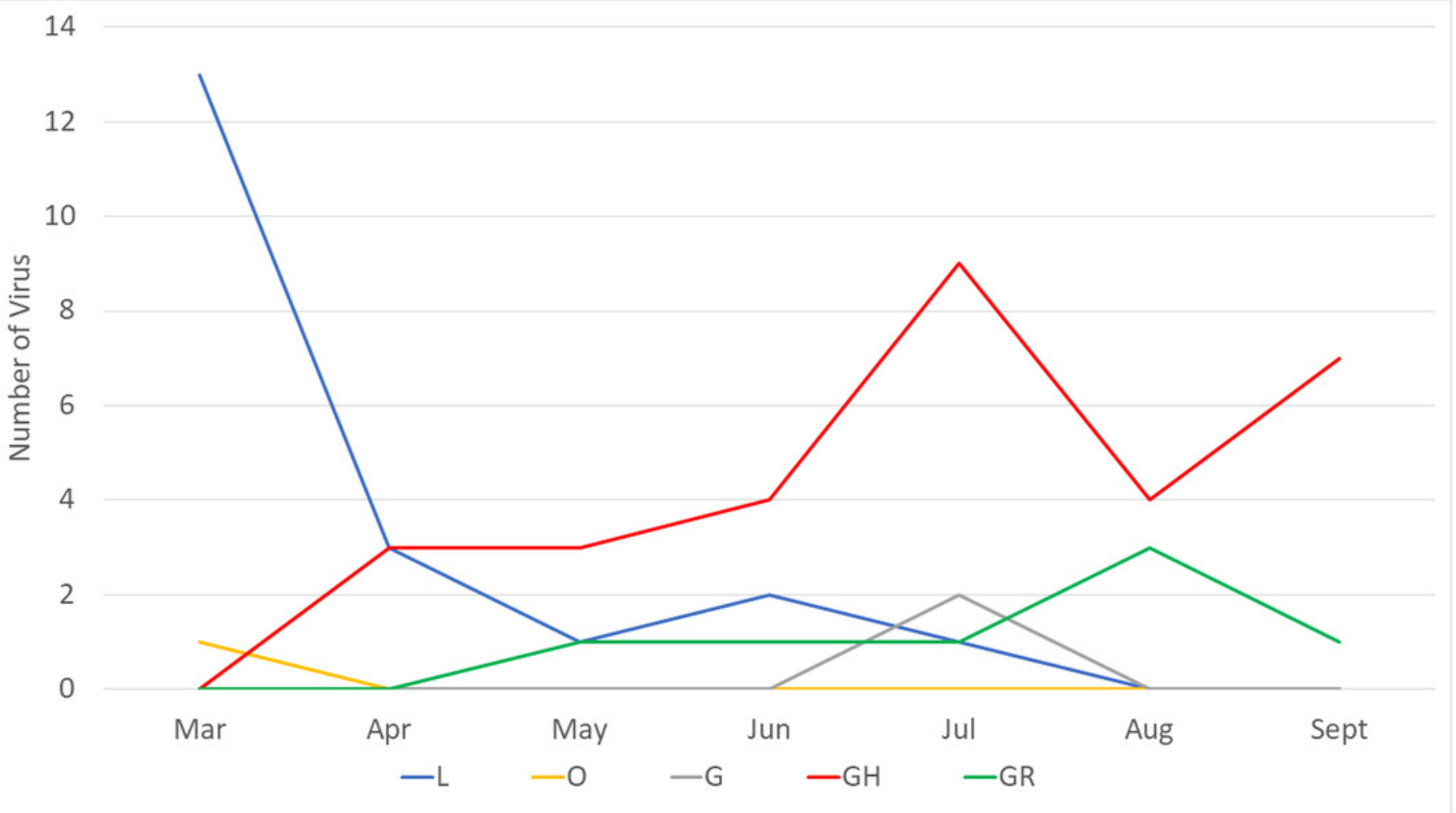




\section{Figure 2}

Phylogenetic analysis of SARS-CoV-2 genomes from Indonesia and different countries.

A phylogenetic tree was constructed from 29.400 nt length of the open reading frame (ORF) of SARS-CoV-2 using the maximum likelihood statistical method with 1,000 bootstrap replications and best-fitting DNA substitution model $(G T R+G+I)$ for the dataset. Virus samples from Yogyakarta and Central Java are indicated in red, while those from other regions in Indonesia are indicated in blue. The tree is drawn to scale $(0.0001)$, with branch lengths measured in the number of substitutions per site. 


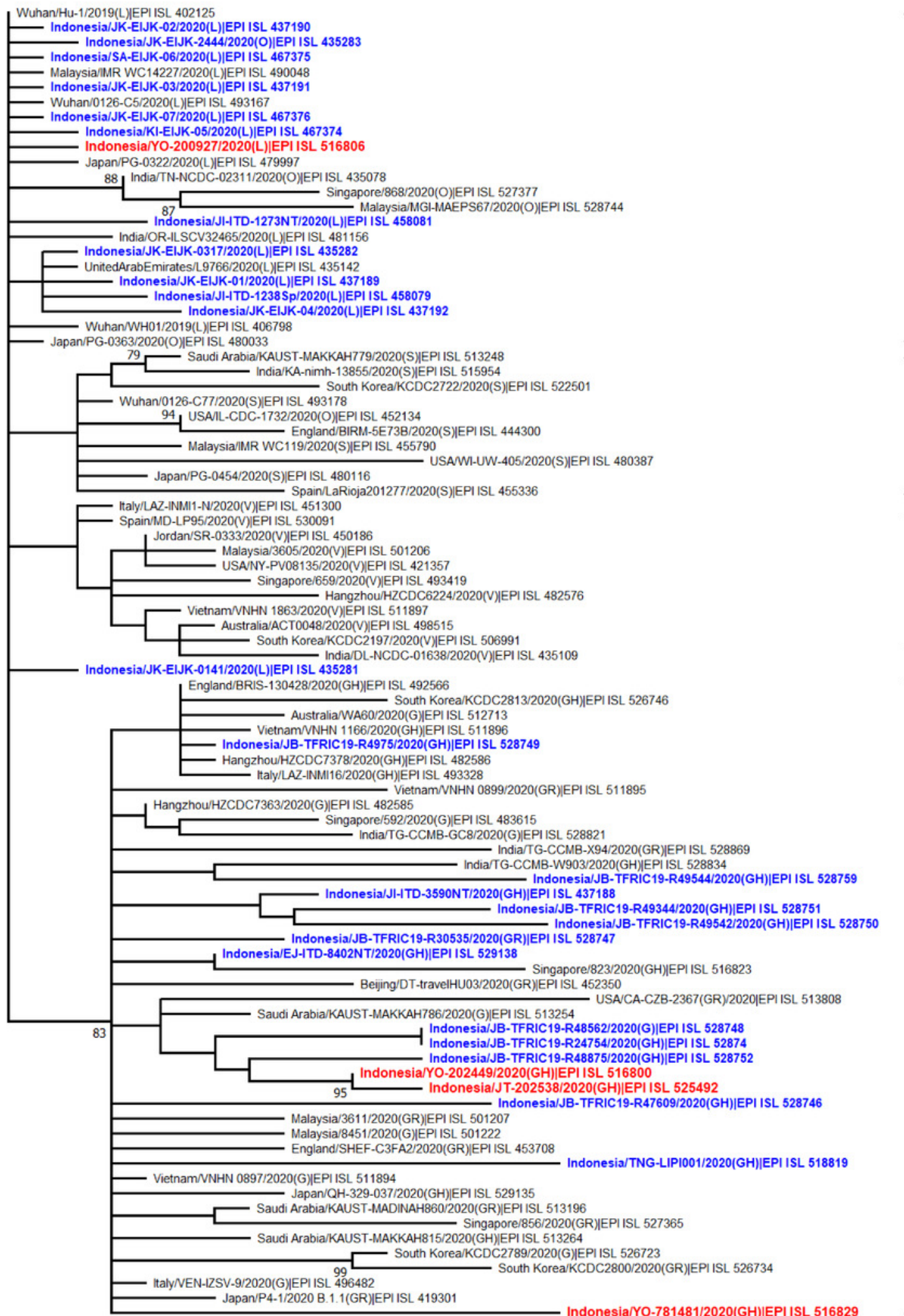

$\mathbf{L} / \mathbf{O}$

$\mathbf{S}$

V

G / GR / GH

\subsection{1}




\section{Table 1 (on next page)}

Characteristics of four patients with COVID-19 and SARS-CoV-2 virus samples from Yogyakarta and Central Java.

* Name of SARS-CoV-2 proteins (bold) are followed by related genes (italic) and amino acid mutation indicated in bracket.

$\mathrm{CT}$, cycle threshold;

Ref. sequence: hCoV-19/Wuhan/Hu-1/2019 (NC_045512.2) 
1 Table 1. Characteristics of four patients with COVID-19 and SARS-CoV-2 virus samples from Yogyakarta and Central Java.

\begin{tabular}{|c|c|c|c|c|c|c|c|c|c|}
\hline $\begin{array}{c}\text { Patient } \\
\text { No }\end{array}$ & Sex & Age (yo) & $\begin{array}{l}\text { COVID-19 } \\
\text { severity }\end{array}$ & $C_{T}$ value & $\begin{array}{c}\text { Virus name } \\
\text { (GISAID Accession ID) }\end{array}$ & $\begin{array}{l}\text { Average } \\
\text { Coverage }\end{array}$ & $\begin{array}{l}\text { Collection } \\
\text { Date }\end{array}$ & $\begin{array}{c}\text { Lineage } \\
\text { (GISAID } \\
\text { clade) }\end{array}$ & $\begin{array}{c}\text { Amino acid mutations* } \\
\text { (no. mutation and position of } \\
\text { proteins-encoded genes) }\end{array}$ \\
\hline 1 & Female & 83 & Moderate & 16.9 & $\begin{array}{l}\text { hCoV19/Indonesia/YO- } \\
\text { UGM-781481/2020 } \\
\text { (EPI_ISL_516829) }\end{array}$ & $3748 x$ & $10 / 08 / 2020$ & GH & $\begin{array}{l}\text { 9: NSP3-ORF1ab (P679S), } \\
\text { NSP12-ORF1ab (P323L, A656S), } \\
\text { NSP13-ORF1ab (M576I), Spike- } \\
S \text { (D614G), NS3-ORF3a (A54V, } \\
\text { Q57H, A99S), NP-N (Q160R) }\end{array}$ \\
\hline 2 & Male & 77 & Moderate & 19.7 & $\begin{array}{l}\text { hCoV19/Indonesia/YO- } \\
\text { UGM-202449/2020 } \\
\text { (EPI_ISL_516800) }\end{array}$ & $22088 x$ & $22 / 06 / 2020$ & GH & $\begin{array}{l}\text { 4: NSP3-ORF1ab (P822L), } \\
\text { NSP12-ORF1ab (P323L), Spike- } \\
S \text { (D614G), NS3-ORF3a (Q57H) }\end{array}$ \\
\hline 3 & Female & 55 & Moderate & 24.7 & $\begin{array}{l}\text { hCov19/Indonesia/JT- } \\
\text { UGM-202538/2020 } \\
\text { (EPI_ISL_525492) }\end{array}$ & $347 x$ & $26 / 06 / 2020$ & GH & $\begin{array}{l}\text { 5: NSP3-ORF1ab (P822L), } \\
\text { NSP12-ORF1ab (P323L), Spike- } \\
S \text { (D614G), NS3-ORF3a (Q57H), } \\
\text { NS7a-ORF7a (H73Y) }\end{array}$ \\
\hline 4 & Male & 30 & Mild & 27.9 & $\begin{array}{l}\text { hCoV19/Indonesia/YO- } \\
\text { UGM-200927/2020 } \\
\text { (EPI_ISL_516806) }\end{array}$ & $102 x$ & $16 / 05 / 2020$ & $\mathrm{~L}$ & 1: NSP5-ORFlab (M49I) \\
\hline
\end{tabular}

2

3 * Name of SARS-CoV-2 proteins (bold) are followed by related genes (italic) and amino acid mutation indicated in bracket.

4 CT, cycle threshold;

5 Ref. sequence: hCoV-19/Wuhan/Hu-1/2019 (NC_045512.2) 


\section{Table 2 (on next page)}

The genome-wide SNPs of four SARS-CoV-2 virus samples from Yogyakarta and Central Java (positions referred to the reference sequence: NC_045512.2).

Name of SARS-CoV-2 proteins (bold) are followed by related genes (italic) and nucleotide variations are colour-shaded

SNPs are shown according to nucleotide positions starting from 5'-UTR (untranslated region) of SARS-CoV-2 genome 
2 Table 2. The genome-wide SNPs of four SARS-CoV-2 virus samples from Yogyakarta and Central Java (positions referred to the 3 reference sequence: NC_045512.2)

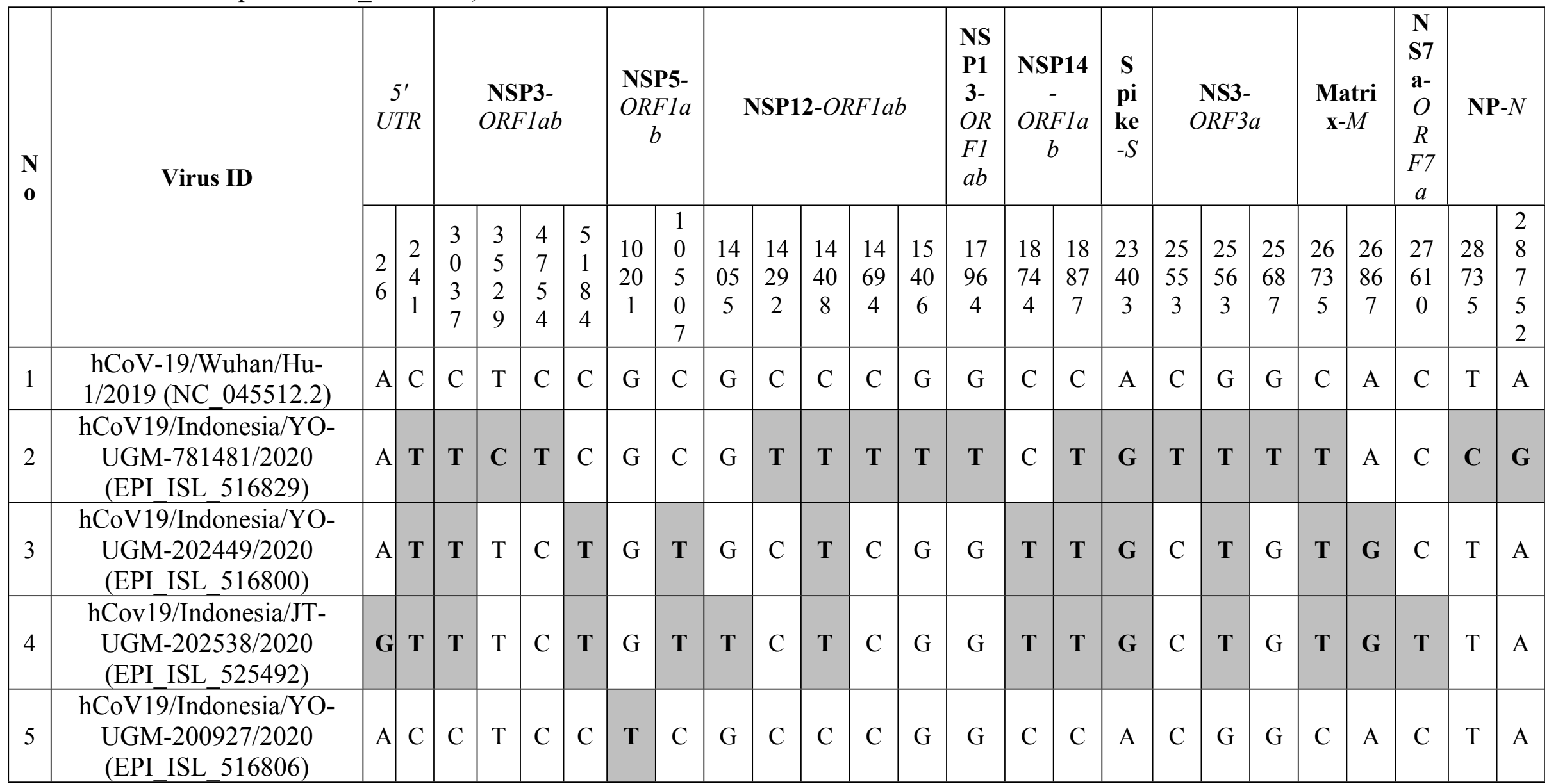

NarAe of SARS-CoV-2 proteins (bold) are followed by related genes (italic) and nucleotide variations are colour-shaded SNBs are shown according to nucleotide positions starting from 5'-UTR (untranslated region) of SARS-CoV-2 genome 


\section{Table 3(on next page)}

The amino acid variations of four SARS-CoV-2 virus samples from Yogyakarta and Central Java (positions referred to the reference sequence: NC_045512.2).

Name of SARS-CoV-2 proteins (bold) are followed by related genes (italic) and amino acid variations are colour-shaded

Amino acid variations are shown starting from translated region of each protein. 
1 Table 3. The amino acid variations of four SARS-CoV-2 virus samples from Yogyakarta and Central Java (positions referred to the 2 reference sequence: NC_045512.2).

3

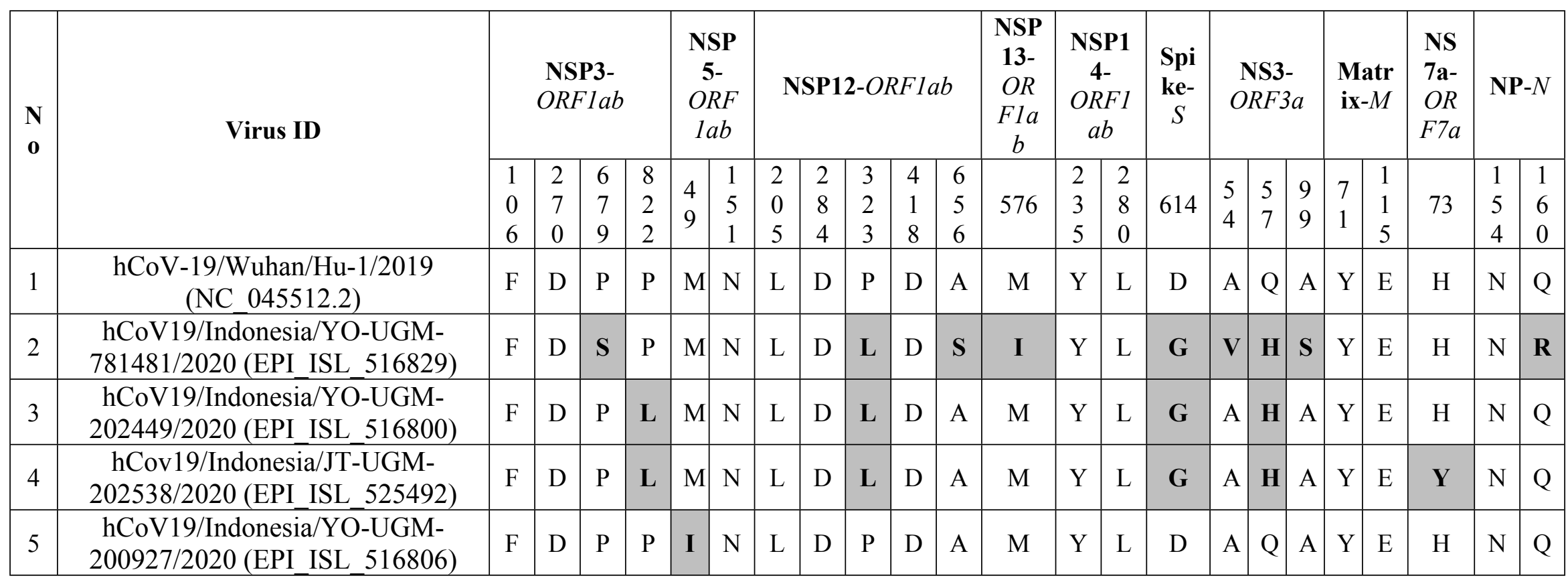

Xame of SARS-CoV-2 proteins (bold) are followed by related genes (italic) and amino acid variations are colour-shaded Amino acid variations are shown starting from translated region of each protein. 